\title{
Self-Consistent Approach in the Microdynamics Description of Supercooled Liquids and Glasses ${ }^{1}$
}

\author{
A. V. Mokshin ${ }^{a}$, R. M. Yulmetyev ${ }^{a}$, R. M. Khusnutdinov ${ }^{a}$, and P. Hänggi ${ }^{b}$ \\ ${ }^{a}$ Tatar State Humanitarian-Pedagogical University, Kazan, 420021 Russia \\ ${ }^{b}$ Department of Physics, University of Augsburg, Augsburg, D-86135 Germany \\ e-mail:mav@theory.kazan-spu.ru \\ Received December 13, 2005
}

\begin{abstract}
A study of the microdynamics of supercooled liquids and glasses is executed through calculations of the dynamic structure factor $S(k, \omega)$. The theory developed on the basis of a self-consistent approach in the framework of the memory function formalism is applied to define the frequency spectra $\left(m / k_{\beta} T\right) S(k, \omega)$ of supercooled argon at the temperature $T=5 \mathrm{~K}$ for the wavenumber region from 2 to $8.5 \mathrm{~nm}^{-1}$. The results obtained are in good agreement with the molecular dynamics simulation data.
\end{abstract}

PACS numbers: 62.60.+v; 47.11.-j; 62.10.+s; 61.20.Gy

DOI: $10.1134 / \mathrm{S} 1063783406090253$

The understanding of the microscopic dynamics of supercooled liquids and of its relation with the glass transition is one of the problems that remain open in the physics of the condensed matter, which has led to the performance of numerous experimental [1-3] and theoretical investigations [4]. Although different details of the microdynamics of supercooled liquids and glasses have been essentially exactly determined, much remains unclear even now. So, for example, even though the relation between the phenomenology of glass transition and the long-time dynamics has been almost clarified, the effect of "the structural arrest" on the high-frequency collective vibrational motion and the role of memory effects in structural relaxation is much less clear [5-7]. The present paper is devoted to the study of this issue.

The most convenient way to study the dynamics of density fluctuations is to determine the dynamic structure factor $S(k, \omega)$, which can be experimentally obtained by means of the scattering of light, neutrons, and $x$ rays. One of the common features established for glass and supercooled liquids via the above-mentioned experimental techniques consists in the fact that acoustic-like excitations in these systems are propagated up to a value of the wavenumber $k$ corresponding to the interparticle distances. As this takes place, the broadening of high-frequency peaks corresponding to these collective excitations follows a power law $D k^{2}$, where $D$ is practically independent of temperature. We need to note that similar features were earlier established in the microdynamics of density fluctuations in liquid alkali metals [8-10]. From the theoretical point of view, $S(k$, $\omega)$ can be found from the generalized Langevin equa-

\footnotetext{
${ }^{1}$ The text was submitted by the authors in English.
}

tion [11] for the normalized density correlator $\phi(t)=$ $\left\langle\delta \rho_{k}(t) \delta \rho_{-k}(0)\right\rangle /\left\langle\left.\delta \rho_{k}(0)\right|^{2}\right\rangle$

$$
\begin{gathered}
\frac{d^{2} \phi(k, t)}{d t^{2}}+\Omega_{1}^{2}(k) \phi(k, t) \\
+\Omega_{2}^{2}(k) \int_{0}^{t} d \tau M_{2}(k, t-\tau) \frac{d \phi(k, \tau)}{d \tau}=0,
\end{gathered}
$$

where $M_{2}(k, t)$ is the second-order memory function, $\Omega_{1}^{2}(k)$ and $\Omega_{2}^{2}(k)$ are the frequency relaxation parameters, which are expressed through the even frequency moments of $S(k, \omega)$,

$$
\omega^{(n)}(k)=\frac{\int d \omega \omega^{n} S(k, \omega)}{\int d \omega S(k, \omega)} .
$$

Namely,

$$
\begin{gathered}
\Omega_{1}^{2}(k)=\omega^{(2)}(k)=\frac{k_{B} T}{m} \frac{k^{2}}{S(k)}, \\
\Omega_{2}^{2}(k)=\frac{\omega^{(4)}(k)}{\omega^{(2)}(k)}-\omega^{(2)}(k) .
\end{gathered}
$$

As was recently shown (see [7], and [12, Eq. (7)]), the Laplace transform, $\tilde{f}(s)=\int_{0}^{\infty} e^{-s t} f(t) d t$, of the nonMarkovian equation (1) for the case of nonergodicity glass systems allows one to obtain the dynamic structure factor in the following form: 

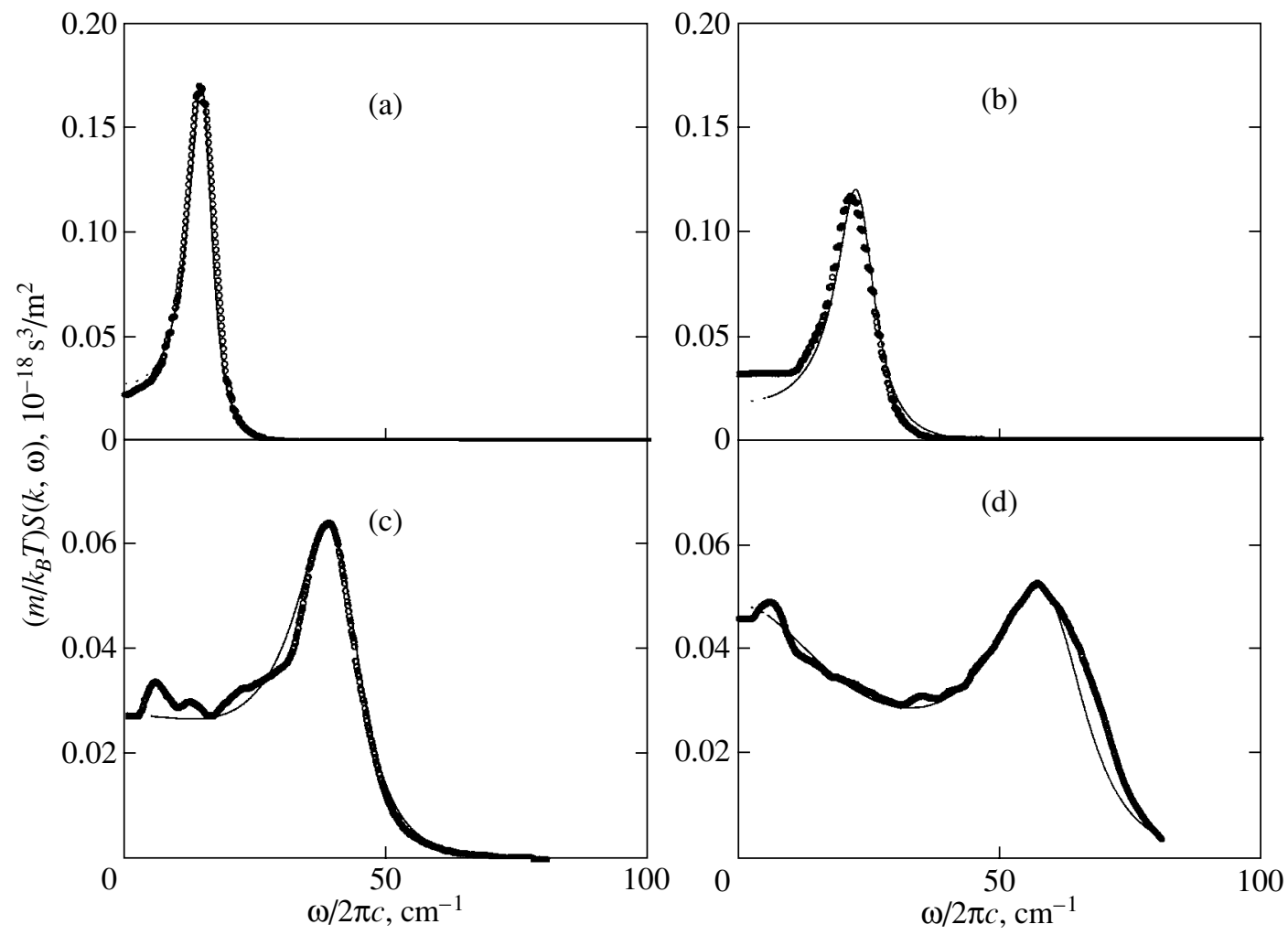

Fig. 1. Frequency spectra of $\left(m / k_{B} T\right) S(k, \omega)$ of supercooled argon for $T=5 \mathrm{~K}$ at $k=$ (a) 2.0 , (b) 2.9 , (c) 5.0 , and (d) $8.5 \mathrm{~nm}^{-1}$. Circles are the data from molecular dynamics simulations [6], and the solid line is our theoretical results.

$$
S(k, \omega)=S(k)\left[f(k) \delta(\omega)+\frac{1-f(k)}{\pi} \frac{\left[\Omega_{1}^{2}(k)+\Omega_{2}^{2}(k)\right] M_{2}^{\prime}(k, \omega)}{\left[\omega^{2}-\Omega_{1}^{2}(k)+\omega \Omega_{2}^{2}(k) M_{2}^{\prime \prime}(k, \omega)\right]^{2}+\left[\omega \Omega_{2}^{2}(k) M_{2}^{\prime}(k, \omega)\right]^{2}}\right],
$$

where $\tilde{M}_{2}(k, i \omega)=M_{2}^{\prime}(k, \omega)+i M_{2}^{\prime \prime}(k, \omega) ; S(k)=$ $\left\langle\left|\delta \rho_{k}(0)\right|^{2}\right\rangle$ is the static structure factor; and $f(k)$ is the nonergodicity factor, which is expressed thorough $\Omega_{1}^{2}(k)$ and $\Omega_{2}^{2}(k)[4,6]$ :

$$
f(k)=\lim _{t \rightarrow \infty} \phi(k, t)=\mathscr{F}\left(\Omega_{1}^{2}(k), \Omega_{2}^{2}(k)\right) .
$$

Then, the problem of defining the dynamic structure factor $S(k, \omega)$ is reduced to finding the second-order memory function $M_{2}(k, t)$ (or its Laplace transform), which is also the time correlation function describing the corresponding relaxation process. From the point of view of the Zwanzig-Mori formalism [13, 14], the Laplace transforms of the whole set of memory functions arising in a hierarchical chain of non-Markovian equations are interrelated by the following recurrent relation:

$$
\tilde{M}_{n}(k, s)=\left[s+\Omega_{n+1}^{2}(k) \tilde{M}_{n+1}(k, s)\right]^{-1}
$$

where $\Omega_{n}^{2}(k)$ is the relaxation parameter of the $n$ order. The finding of the term $M_{2}(k, t)$ can also be executed in the framework of a self-consistent approach based on an assumption regarding the equalization of time scales of high-order memory functions, $\tau_{3}(k)$ and $\tau_{4}(k)$, where $\tau_{n}(k)=\int_{0}^{\infty} d t M_{n}(k, t)[8] .{ }^{2}$ As a result, we obtain the termination in the recurrent relation (7) and find exactly the following expression for $M_{2}(k, t)$ without any trivial approximations for the memory function $M_{2}(k, t)$ (or $M_{2}(k, s)$ ) by different model time (frequency) dependences:

$$
\tilde{M}_{2}(k, s)=\left[s+\frac{\Omega_{3}^{2}(k) \sqrt{s^{2}+4 \Omega_{4}^{2}(k)}-\Omega_{3}^{2}(k) s}{2 \Omega_{4}^{2}(k)}\right] \text {. }
$$

Now, the spectra $S(k, \omega)$ can be deduced through the simple substitution of Eq. (8) into Eq. (5). So, the posi-

\footnotetext{
2 This interrelation between $\tau_{3}(k)$ and $\tau_{4}(k)$ is based on the assumption the time scales for the TCF of energy current fluctuations and its memory function are equal.
} 


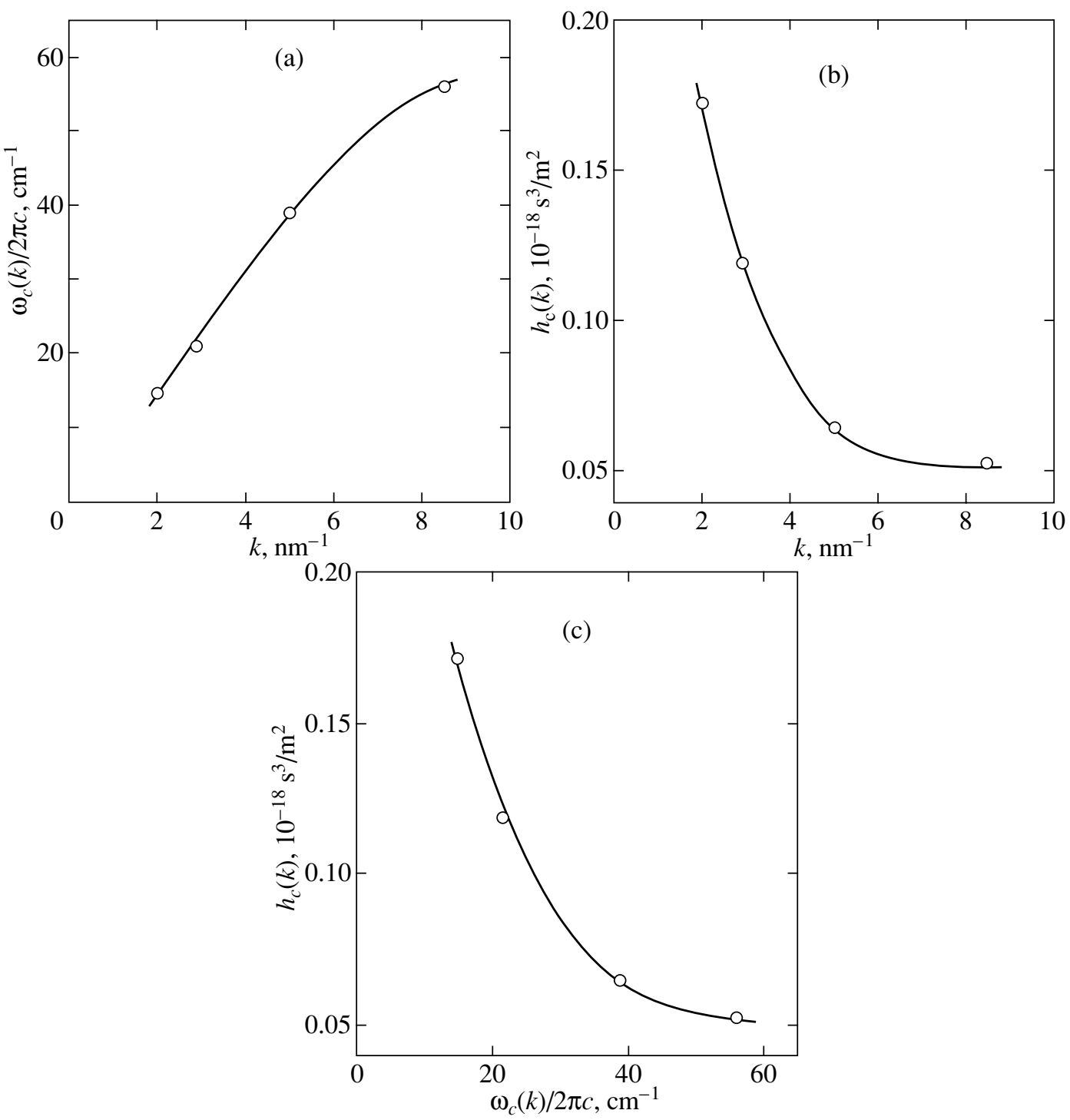

Fig. 2. (a) Dispersion of the side peak of the dynamic structure factor for supercooled argon $(T=5 \mathrm{~K})$, (b) $k$ dependence of the side peak amplitude, and (c) dependence of the side peak amplitude $h_{c}$ on the frequency of collective excitations $\omega_{c} / 2 \pi c$. The solid line represents theoretical results, and circles are the data from molecular dynamics simulations [6].

tion, as well as the broadening, and the amplitude of the high-frequency peak of $S(k, \omega)$ in this approach are interrelated terms determined by the frequency relaxation parameters $\Omega_{3}^{2}(k)$ and $\Omega_{4}^{2}(k)$. We need to note that, as was recently shown in [15], this approach allows one to obtain the second-order memory function in terms of simple relaxation functions. This is in full agreement with the concepts of mode-coupling theory [16]. In accordance with the presented approach, the spectra of the dynamic structure factor $S(k, \omega)$ were calculated by Eqs. (5) and (8) for supercooled argon at a temperature $T=5 \mathrm{~K}$ for wavenumbers $k=2.0,2.9,5.0$, and $8.5 \mathrm{~nm}^{-1}$. The parameter $\Omega_{1}^{2}(k)$ was determined exactly from Eq. (3), whereas the numerical values of the second-order relaxation parameter $\Omega_{2}^{2}(k)$ were taken from [6]. The frequency parameters $\Omega_{3}^{2}(k)$ and $\Omega_{4}^{2}(k)$ were determined from a comparison of the theoretical results with the molecular dynamics simulation data. The theoretical results obtained for the reduced dynamical structure factor $\left(m / k_{B} T\right) S(k, \omega)$ (solid line) together with the results of the molecular dynamics simulation (circles) [6] are presented in Fig. 1. We need to note that the molecular dynamics study from [6] was performed for a system of $N=2048$ argon atoms interacting via a Lennard-Jones potential $\left(\epsilon / k_{B}=125.2 \mathrm{~K}\right.$, $\sigma=3.405 \AA$ ). It is obvious that the theoretical curves are in good agreement with the molecular dynamics data for the whole range of wavenumber values. The insignificant oscillations observed in the data from 


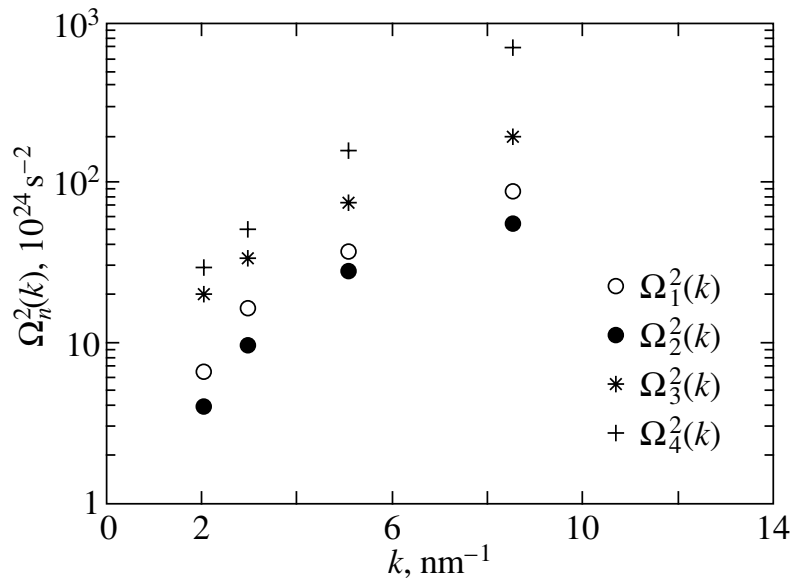

Fig. 3. Frequency relaxation parameters $\Omega_{n}^{2}(k), n=1,2,3$, and 4 used in the theoretical analysis of $S(k, \omega)$ for supercooled argon at $T=5 \mathrm{~K}$.

molecular dynamics simulations for the low-frequency regions of the dynamic structure factor spectra are related to errors that arise at the numerical Laplace (Fourier) transform of data for the density correlator [6]. Good agreement between theory and the molecular dynamics simulation data are also seen in Fig. 2, where dispersion of the high-frequency peak of the dynamic structure factor (see Fig. 2a) and the dependences of the side peak amplitude $h_{c}$ on the wavenumber $k$ (see Fig. 2b) and on the frequency $\omega_{c} / 2 \pi c$ at fixed values of $k$ (see Fig. 2c) are presented. In Fig. 3, we present the numerical values of the frequency relaxation parameters $\Omega_{n}^{2}(k), n=1,2,3$, and 4 used in our calculations. We need to note here that all frequency relaxation parameters have the same $k$ dependence. A similar scenario was found earlier for the description of the microscopic dynamics of liquid alkali metals (lithium, sodium, rubidium, cesium) near their melting temperatures $[8-10,15]$.

In conclusion, this paper is devoted to the development of a self-consistent approach executed in the framework of the memory function formalism and suggested earlier for description of the microdynamics of liquid alkali metals for finding the dynamic structure factor of supercooled liquids. The results of the theoretical analysis of $S(k, \omega)$ performed for supercooled argon at the temperature $T=5 \mathrm{~K}$ for wavenumbers values ranging from 2.0 to $8.5 \mathrm{~nm}^{-1}$ are in good agreement with the data from molecular dynamics simulation. This allows us to make the following inferences.

(1) It is possible to use the quasi-hydrodynamic approach for the description of "instantaneous" dynamical processes in supercooled liquids and glasses on a time scale of $10^{-12} \mathrm{~s}$ in microscopical spaces.
(2) Microscopic processes and the corresponding collective excitations observed in the terahertz frequency region of the dynamic structure factor spectra have a common origin in liquid alkali metals, as well as in supercooled liquids, which can serve as convincing proof of the benefit of the assumptions made in [17].

\section{ACKNOWLEDGMENTS}

The authors are grateful to V. Götze, M. Sperl, and V.Y. Shurygin for useful correspondence regarding the study.

This work was partially supported by the Russian Ministry of Education and Science (grant no. 03-0600218a) and Russian Foundation for Basic Research (no. 02-02-16146).

\section{REFERENCES}

1. A. Matic, L. Börjesson, G. Ruocco, C. Masciovecchio, A. Mermet, F. Sette, and R. Verbeni, Europhys. Lett. 54 (1), 77 (2001).

2. C. Masciovecchio, G. Monaco, G. Ruocco, F. Sette, A. Cunsolo, M. Krisch, A. Mermet, M. Soltwisch, and R. Verbeni, Phys. Rev. Lett. 80, 544 (1998).

3. D. Fioretto, U. Buchenau, L. Comez, A. Sokolov, C. Masciovecchio, A. Mermet, G. Ruocco, F. Sette, L. Willner, B. Frick, D. Richter, and L. Verdini, Phys. Rev. E: Stat. Phys., Plasmas, Fluids, Relat. Interdiscip. Top. 59, 4470 (1999).

4. W. Götze, in Liquids, Freezing, and the Glass Transition, Ed. by J. P. Hansen, D. Levesque, and J. Zinn-Justin (North-Holland, Amsterdam, 1991).

5. A. V. Mokshin, R. M. Yulmetyev, and P. Hänggi, Phys. Rev. Lett. 95, 200601 (2005).

6. G. Ruocco, F. Sette, R. Di Leonardo, G. Monaco, M. Sampoli, T. Scopigno, and G. Viliani, Phys. Rev. Lett. 84, 5788 (2000).

7. G. Ruocco and F. Sette, J. Phys.: Condens. Matter 13, 9141 (2001).

8. R. M. Yulmetyev, A. V. Mokshin, P. Hänggi, and V. Yu. Shurygin, Phys. Rev. E: Stat., Nonlinear, Soft Matter Phys. 64, 057101 (2001).

9. R. M. Yulmetyev, A. V. Mokshin, P. Hänggi, and V. Yu. Shurygin, Pis'ma Zh. Éksp. Teor. Fiz. 76 (3), 181 (2002) [JETP Lett. 76 (3), 147 (2002)].

10. R. M. Yulmetyev, A. V. Mokshin, T. Scopigno, and P. Hänggi, J. Phys.: Condens. Matter 15, 2235 (2003).

11. U. Balucani and M. Zoppi, Dynamics of the Liquid State (Clarendon, Oxford, 1994).

12. S. Mossa, G. Monaco, G. Ruocco, M. Sampoli, and F. Sette, J. Chem. Phys. 116, 1077 (2002).

13. R. Zwanzig, Phys. Rev. 124, 1338 (1961).

14. H. Mori, Prog. Theor. Phys. 33, 423 (1965).

15. A. V. Mokshin, R. M. Yulmetyev, and P. Hänggi, J. Chem. Phys. 121, 7341 (2004).

16. S. P. Das, Rev. Mod. Phys. 76, 785 (2004).

17. T. Scopigno, G. Ruocco, F. Sette, and G. Monaco, Science (Washington) 302, 849 (2003). 